\title{
A RADIOGRAPHIC EVALUATION OF MANDIBULAR ASYMMETRY IN CLASS II DIVISION 1 MALOCCLUSION PATTERN
}

\author{
Dr. Manish Bajracharya, ${ }^{1}$ Dr. Praveen Mishra, ${ }^{2}$ Dr. Prakash Bhattarai, ${ }^{3}$ Dr. Umesh Parajuli, ${ }^{4}$ \\ Dr. Reetu Shrestha, ${ }^{5}$ Dr. Keshab Raj Poudel ${ }^{6}$ \\ 1. Assistant Professor, Nepal Army Institute of Health Sciences, Kathmandu \\ 2. Professor, National Academy Medical Science, Kathmandu \\ 3. Associat Professor, Nepal Medical College, Collage of Dental Surgery, Kathmandu \\ 4. Lecturer, Gandaki Medical College, Pokhara \\ 5. Lecturer, Kathmandu University School of Medical Sciences, Dhulikhel \\ 6. Assistant Professor, Kathmandu Medical College, Kathmandu
}

Correspondence : manishbajracharya@gmail.com

\section{ABSTRACT}

Introduction: Mandibular asymmetry may cause not only esthetic problem but also functional problem as it has a direct role in stomatognathic system.

Objective: The objective of this study was to find out the difference between condylar asymmetry ratio in Class II division 1 malocclusion group and normal occlusion group in males and females.

Materials \& Method: Orthopantomogram radiographs were selected from the pool of records such that there were 40 Class II division 1 males and females above 20 years of age. Thirty normal occlusion subjects ( 15 males, 15 females) were enrolled and orthopantomogram records were taken. All the films were hand traced on the acetate paper and the mean condylar, ramal and condylar plus ramal asymmetry index were measured in the normal and Class II Division 1 groups according to the method given by Habets. Independent sample t-test was done to compare between the normal occlusion and Class II Division 1 malocclusion for each male and female group.

Result: Significant difference was observed between condylar heights, combined height and condylar asymmetrical ratio. Respectively in females. However, all other asymmetrical ratios were insignificant. Similarly, for males, condylar height, ramus height, combined (condyle and ramus) were all significant respectively. However, the asymmetric ratios were found to be insignificant. There is a significant difference between Class II division 1 malocclusion and normal occlusion group in terms of condylar asymmetry ratio in female subjects. However, it was not significant in case of male subjects.

Conclusion: Our study suggests that females with Class II division 1 malocclusion more prone to TMD in comparison to males.

Key words: Class II Division 1, condylar asymmetry, orthopantomogram

\section{INTRODUCTION:}

Orthopantomogram (OPG) is one of the most useful radiographs in dentistry. Panaromic radiography is frequently used in the orthodontic practice to provide important information about the teeth, their axial inclinations, maturation periods and surrounding tissues. It is also taken into consideration for assessing the condyle and symmetry of the mandible on the right and left side. When dealing with facial morphology, symmetry refers to the correspondence in size, shape, and location of facial landmarks on the opposite sides of the median sagittal plane ${ }^{1}$. Symmetries of the mandible is utmost important from esthetic and functional point of view. Asymmetries of the mandible may lead to functional problems because of its role in the stomatognathic system. Condylar cartilage is considered to have the highest growth potential. Any injury to the condyle area especially during the growing phase can alter the growth potential of the cartilage leading to displacement of the mandible towards the affected side. Thus, condylar asymmetries are thought to be one of the most important causes of mandibulofacial asymmetries ${ }^{2-4}$. Asymmetry in the face as well as dentition within normal limits is a naturally occurring phenomenon. So there is a dilemma of whether different malocclusions caused by dentoalveolar or skeletal deviations or any compromise treatment plans lead to additional complications, such as tipping of the occlusal plane, dental instability, or temporomandibular disharmonies ${ }^{5}$. The relationships between the condylar asymmetries and craniomandibular disorders were investigated by Habets and his coworkers ${ }^{6}$. Kjellberg et $\mathrm{al}^{7}$ developed and used a new method of quantitatively measuring the effects of condylar heights on panoramic radiographs. Panoramic radiography is relatively accessible, provides a bilateral view of the mandible, cost less and generally taken before orthodontic treatment. This study aims to find out if there is any condylar asymmetry in Class II division 1 malocclusions in comparison to normal occlusion. 


\section{MATERIALS AND METHOD:}

The study was done in Orthodontic Unit, Dental Department, National Academy of Medical Sciences, Bir Hospital. Two groups were divided consisting of 80 Class II Division 1 malocclusion (40 males, 40 females) and 30 normal occlusion subjects (15 males, 15 females). The normal occlusion group consisted of subjects of age group above 20 years; full complement of teeth present except the third molars; Class I molar and canine relation; normal over jet and overbite; minor or no crowding; normal growth and development with good alignment of upper and lower arches; good facial symmetry determined clinically; no significant medical history; no history of facial trauma, no previous orthodontic, prosthodontic or maxillofacial or plastic surgery; no signs and symptoms of TMJ dysfunction. Similarly for Class II Division 1 malocclusion case consisted of subjects with age group above 20 years; full cusp class II with overjet more than $5 \mathrm{~mm}$; full complement of teeth with the exception of third molars; no history of previous orthodontic and prosthodontic treatment; no any history of facial trauma; no any congenital and dentofacial abnormalities present; a good quality OPG radiograph; no any signs and symptoms of TMJ dysfunction.

Intraoral photographs and plaster models were used to classify patients according to their malocclusion. Two operators evaluated the subjects clinically for determining the fulfillment of the inclusion criteria of the selected samples. All the OPG radiographs classified as class II patients were collected from the records in the Dental department. Radiographs of the patients meeting the inclusion criteria were randomly selected such that there will be equal number of males and females of 40 each. For the normal occlusion group, 15 males and 15 females each ranging from 20 to 32 years of age were enrolled. Orthopantomogram radiographs were taken after their verbal and informed consent. The radiographic machine used for all the patients was Rotaplus model 9308525XOY with maximum exposure of 17 seconds. The line frequency was $50 \mathrm{~Hz}$. The mean enlargement of the image was 1.2:1. The inherent filtration of the PAN cassette was $1 \mathrm{~mm} \mathrm{Al} \mathrm{eq} \mathrm{at} 70 \mathrm{Kvp}$. All the radiographs were taken by the same operator to avoid interoperator variation. The OPG was taken with lip in relaxed position and the head oriented to the Natural head position.

All the films were hand traced by the same operator on the acetate paper and the mean condylar, ramal and condylar plus ramal asymmetry index were measured in the normal and Class II Division 1 groups according to the method given by Habets et $\mathrm{al}^{8}$. The landmarks are shown in Figure 1. The borders of the condyle, neck, ramus and corpus of both sides of the mandible were traced. A line was drawn such that it is tangent to the ramus (Line A) and that it contacted the most lateral points on the condyle (L1) and the ascending ramus (L2). To the tangent of the ramus (Line A), a perpendicular line was drawn such that it passed through the most superior point of the condyle (Line B). The perpendicular distance between $\mathrm{L} 1$ and Line B was called the condylar height $(\mathrm{CH})$. The distance between $L 1$ and $L 2$ was called the ramus height (RH). All the measurements were obtained with the help of vernier caliper (Mitutoyo SER No. 60325791 Japan) for linear which recorded up to $0.02 \mathrm{~mm}$. To measure the asymmetry index the following formula was used:

Asymmetry index $=\mathrm{CH}$ right- $\mathrm{CH}$ left $\times 100$

$\mathrm{CH}$ right $+\mathrm{CH}$ left

One month after the first measurements, 10 OPGs from the normal group and 20 OPGs from the study group were randomly selected and reexamined by the same operator. Dalburgs formula was applied to check for intraexaminer variation in the measurement. The value obtained was 0.51 to 0.92 . The scores and grouping criteria were entered into a SPSS software package version 17.0. Independent sample t-test was done to compare between the normal occlusion and Class II Division 1 malocclusion for each male and female groups.

Landmarks:

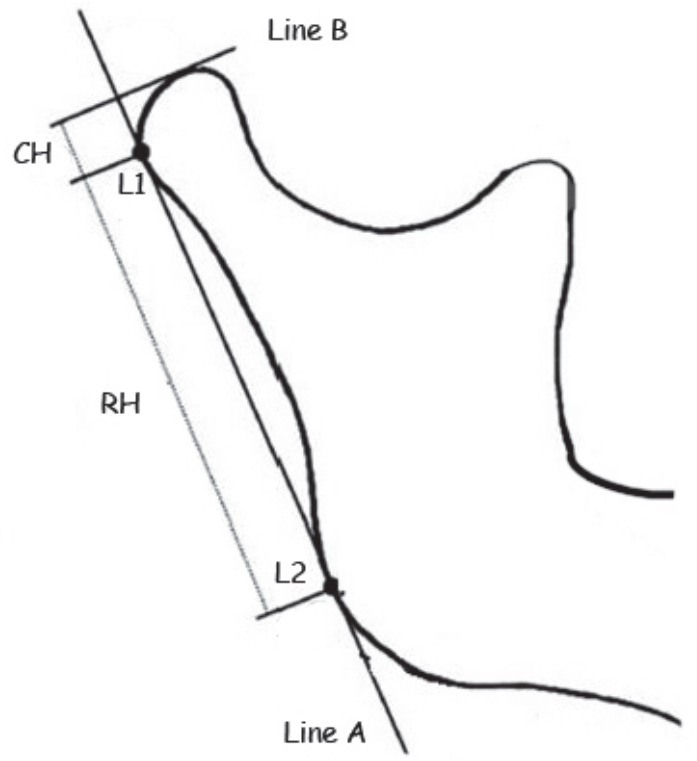

Figure 1: Measurements of the asymmetry index according to Habets et al.

(L1 and L2: Most lateral points of the tracing of the condyle and ramus of the OPG; Line A: Ramus tangent; Line $B$ : Perpendicular line from $A$ to the most superior part of the condylar image; $\mathrm{CH}$ : Condylar Height; $\mathrm{RH}$ : Ramus Height)

\section{RESULTS:}

The results show that there is significant difference between condylar heights (right and left), combined height (right and left) and condylar asymmetrical ratio. 
( $p=0.000, p=0.000, p=0.010, p=0.003, p=0.049$ ) respectively in females. However, all other asymmetrical ratios are insignificant (Table 1). Similarly, for males, condylar height (right and left), ramus height (right and left), combined (condyle and ramus) are all significant ( $p=0.003, p=0.07, p=0.09, p=0.40, p=0.001, p=0.009$ respectively). However, the asymmetric ratios are all insignificant (Table 2 ).

Table 1: Comparison between normal occlusion and Class II Div 1 (females)

\begin{tabular}{|l|c|c|c|c|c|c|}
\hline \multirow{2}{*}{} & \multicolumn{2}{|c|}{ Normal occlusion } & \multicolumn{2}{c|}{ Class II Div 1} & \multirow{2}{*}{ P Value } & \multirow{2}{*}{ Significance } \\
\cline { 2 - 6 } & Mean & SD & Mean & SD & & \\
\hline Right condylar height & 7.83 & 1.71 & 5.11 & 1.30 & .000 & $* * *$ \\
\hline Left condylar height & 7.06 & 1.66 & 5.00 & 1.33 & .000 & $* * *$ \\
\hline Right ramus height & 49.60 & 3.75 & 48.00 & 5.59 & .317 & NS \\
\hline Left ramus height & 49.66 & 3.84 & 46.95 & 5.50 & .090 & NS \\
\hline Right combined height & 57.43 & 3.88 & 53.11 & 5.68 & .010 & $*$ \\
\hline Left combined height & 56.73 & 3.85 & 51.95 & 5.33 & .003 & $* * *$ \\
\hline Condylar asymmetrical ratio & 7.84 & 7.37 & 8.66 & 8.08 & .049 & $*$ \\
\hline Ramus asymmetrical ratio & 2.47 & 2.30 & 2.55 & 2.65 & .926 & NS \\
\hline Combined asymmetrical ratio & 2.19 & 1.82 & 2.27 & 2.33 & .917 & NS
\end{tabular}

NS indicates Not significant; ${ }^{* * *} p<0.001 ;{ }^{* *} p<0.01 ;{ }^{*} p<0.05$

Table 2: Comparison between normal occlusion and Class II Div 1 (males)

\begin{tabular}{|c|c|c|c|c|c|c|}
\hline & \multicolumn{2}{|c|}{ Normal occlusion } & \multicolumn{2}{|c|}{ Class II Div 1} & \multirow{2}{*}{$P$ Value } & \multirow{2}{*}{ Significance } \\
\hline & Mean & SD & Mean & SD & & \\
\hline Right condylar height & 7.63 & 2.13 & 5.60 & 1.44 & .003 & $* * *$ \\
\hline Left condylar height & 6.96 & 1.75 & 5.46 & 1.10 & .007 & $* *$ \\
\hline Right ramus height & 54.23 & 6.78 & 48.67 & 4.45 & .009 & $* *$ \\
\hline Left ramus height & 52.06 & 5.95 & 48.28 & 4.58 & .040 & $*$ \\
\hline Right combined height & 61.86 & 7.18 & 54.28 & 5.02 & .001 & $* * *$ \\
\hline Left combined height & 59.03 & 6.37 & 53.75 & 4.82 & .009 & $* *$ \\
\hline Condylar asymmetrical ratio & 6.15 & 5.30 & 9.46 & 8.63 & .115 & NS \\
\hline Ramus asymmetrical ratio & 2.89 & 1.71 & 2.51 & 1.86 & .495 & NS \\
\hline Combined asymmetrical ratio & 2.98 & 1.59 & 2.23 & 1.71 & .153 & NS \\
\hline
\end{tabular}

NS indicates Not significant; *** $p<0.001 ;{ }^{* *} p<0.01 ;{ }^{*} p<0.05$

\section{DISCUSSION:}

Generally, asymmetries of the dentofacial complex are assessed in Posterioanterio view of the skull, ${ }^{9}$ submentovertex, ${ }^{10}$ Computed tomography $\operatorname{scan}^{11-13}$ and Magnetic resonance imaging. ${ }^{14}$ Most of the above mentioned techniques cost high and generally not used so commonly during the normal orthodontic treatment. OPGs are one of the most commonly used radiographs and relatively cost less, which are generally taken along with cephalogram for all the patients before commencing an orthodontic treatment. It gives an image of the teeth, its surrounding structures, TMJ and other structures in one exposure. Besides, orthodontic treatment alone can alter only the lower third of the face and all the necessary hard tissues in this area can be viewed in OPG. There is magnification in OPG which is a concern for its accuracy in the measurements. However, studies have shown that there is only slight alteration in vertical measurements unlike the horizontal measurements which allows panoramic radiographs to be used for assessing the vertical asymmetries. ${ }^{15-18}$ So in this study, we have focused only on the vertical measurements and the ratios of the measurements. OPGs have also been used to measure the gonial angles and ramus height of the mandible in complete denture wearer and other anomalies of the mandible. ${ }^{19}$ Habets et $\mathrm{al}^{17}$ have given a technique to determine vertical asymmetric ratio and with this technique it was shown that if there is a difference of more than $6 \%$ between the right and left condyles in OPG measurements, then it indicates a condylar asymmetry.

In this study, we compared the symmetries between the normal occlusion groups and the Class II Div 1 group in both the sexes. It was observed that all the measurement parameters included in this study are less in Class II Div 1 malocclusion group than in the normal occlusion groups. In females, condylar height is decreased in comparison to the normal occlusion group which is statistically significant. The condylar index in Class II Div 1 is increased. Study done by Miller et $\mathrm{al}^{20}$ stated that increase in condylar asymmetry is related to the strong forces that are applied to the articulating surface of the condyle. Due to constant increased force, the articulating surface would increase in surface as an adaptive mechanism. Various 
studies have shown that increase in condylar asymmetry may lead to temporomandibular disorders (TMD)..$^{21-22}$ The research done by Yanez-Vico et al. ${ }^{23}$ using 3D-CT to find the association between condylar asymmetry and TMD found that condylar width, height and length were asymmetrical and was a common feature of TMD.

Studies done with other occlusion types and condylar asymmetries have yield different results. Studies done by Miller and Smidt ${ }^{24}$ regarding the relationship between condylar asymmetries of Class I and Class II Div 2 with deep bite and no signs of TMD showed that there is no any significant difference between the groups. Similarly, Miller et al ${ }^{25}$ did a similar study regarding the relationship between condylar asymmetry of Class I occlusion and Class III malocclusion subjects. They did not find any significant difference between the groups. Similarly, comparison of the condylar asymmetry was done between the unilateral and bilateral crossbites by Veli et al. ${ }^{13}$ The condylar asymmetry was found to be significantly higher. However, Letzer and Kronman ${ }^{26}$ found skeletal asymmetry to be independent of occlusion. However this study was done in posteroanterior film and condylar asymmetries were not taken into consideration.

In males, the difference between the condylar height, ramus height, and combined heights are all statistically significant but the entire asymmetrical index are insignificant. Though in this study, selection of Class II Div 1 subjects without TMD signs and symptoms were done, it seems that females with Class II Div 1 are more prone to TMDs; however more detail research needs to be done regarding it.

\section{CONCLUSION:}

Condylar asymmetry was significantly different in female subjects between Class II division 1 malocclusion and normal occlusion. However, it was not significant in case of male subjects. Our study suggests that females with Class II Div 1 seem to be related with condylar asymmetry and thus more prone to TMD in comparison to males.

\section{REFERENCES}

1. Peck S, Peck L, Kataja M. Skeletal asymmetry in esthetically pleasing faces. Angle Orthod 1991;61(1):43-8.

2. Schellhas KP, Piper AJ, Omlie MR. Facial skeleton remodeling due to temporomandibular joint degeneration: an imaging study of 100 patients. Am J Neuroradiol 1990;11:541-51.

3. Westesson PL, Tallents TL, Katzberg RW, Guay JA. Radiographic assessment of asymmetry of the mandible Am J Neuroradiol 1994;15:991-99.

4. Yamashiro T, Okada T, Takada K. Case report: facial asymmetry and early condylar fracture. Angle Orthod 1998;68(1):85-90.

5. Turpin DL. Correcting the Class II subdivision malocclusion. Am J Orthod Dentofacial Orthop 2005;128(5):555-6.

6. Habets LL, Bezuur JN, Naeiji M, Hanson TL. The orthopantomogram as an aid in diagnosis of Termporomandibular problems II. The vertical symmetry. J Oral Rehabilitation 1988(15):465-71.

7. Kjelberg H, Ekestubbe A, Kiliaridis S, Thilander B. Condylar height on panoramic radiographs. A methodological study with a clinical application. Acta odontol Scand 1994(52):43-50.

8. Habets LL, Bezuur JN, Naeiji M, Hansson TL. The Orthopantomogram, an aid in diagnosis of temporomandibular joint problems. II. The vertical symmetry. J Oral Rehabil 1988;15(5):465-71.

9. Kambylafkas P, Kyrkanides S, Tallents RH. Mandibular asymmetry in adult patients with unilateral degenerative joint disease. Angle Orthod 2005;75(3):305-10.

10. Rose JM, Sadowsky C, BeGole EA, Moles R. Mandibular skeletal and dental asymmetry in Class II subdivision malocclusions. Am J Orthod Dentofacial Orthop 1994;105(5):489-95.

11. Vitral RW, Telles Cde S. Computed tomography evaluation of temporomandibular joint alterations in class II Division 1 subdivision patients: condylar symmetry. Am J Orthod Dentofacial Orthop 2002;121(4):369-75.

12. Lee H, Bayome M, Kim SH, Kim KB, Behrents RG, Kook YA. Mandibular dimensions of subjects with asymmetric skeletal class III malocclusion and normal occlusion compared with cone-beam computed tomography. Am J Orthod Dentofacial Orthop 2012;142(2):179-85.

13. Veli I, Uysal T, Ozer TU, F.I. , Eruz M. Mandibular asymmetry in unilateral and bilateral posterior crossbite patients using conebeam computed tomography. Angle Orthod 2011 81(6):966-74.

14. Westesson PL, Tallents RH, Katzberg RW, Guay JA. Radiographic assessment of asymmetry of the mandible. Am J Neuroradiol 1994;15(5):991-9.

15. Habets LLMH, Bezuur JN, Naeiji M, Hansson TL. The orthopantomograph, an aid in diagnosis of temporomandibular joint problems. II. The vertical symmetry. J Oral Rehabil 1988(15):465-71.

16. Larheim TA, Svanaes DB. Reproducibility of rotational panoramic radiography: mandibular linear dimensions and angles. Am J Orthod Dentofacial Orthop 1986;90(1):45-51. 
17. Habets LL, Bezuur JN, van Ooij CP, Hansson TL. The orthopantomogram, an aid in diagnosis of temporomandibular joint problems. I. The factor of vertical magnification. J Oral Rehabil 1987;14(5):475-80.

18. Kambylafkas P, Murdock E, Gilda E, Tallents RH, Kyrkanides S. Validity of panoramic radiographs for measuring mandibular asymmetry. Angle Orthod 2006;76(3):388-93.

19. Miller VJ, Myers SL, Zeltser C, Yoeli Z. The relation of age and handedness to condylar asymmetry in a group of edentulous patients with a temporomandibular disorder of arthrogenous origin. J Oral Rehabil 1995;22(4):311-3.

20. Miller VJ, Yoeli Z, Barnea E, Zeltser C. The effect of parafunction on condylar asymmetry in patients with temporomandibular disorders. 1998(25):721-4.

21. Saglam AA, Sanli G. Condylar asymmetry measurements in patients with temporomandibular disorders. J Contemp Dent Pract 2004;5(3):59-65.

22. Miller VJ, Yoeli Z, Barnea E, Zeltser C. The effect of parafunction on condylar asymmetry in patients with temporomandibular disorders. J Oral Rehabil 1998;25(9):721-4.

23. Yáñez-Vico RM, Iglesias-Linares A, Torres-Lagares D, Gutiérrez-Pérez JL, Solano-Reina E. Association between condylar asymmetry and temporomandibular disorders using 3D-CT. Med Oral Patol Oral Cir Bucal 2012.;17(5):e852-8.

24. Miller VJ, Smidt A. Condylar asymmetry and age in patients with an Angle's Class II division 2 malocclusion. J Oral Rehabil 1996;23(10):712-5.

25. Miller VJ, Bodner L. Condylar asymmetry measurements in patients with an Angle's Class III malocclusion. J Oral Rehabil 1997;24(3):247-9.

26. Letzer GM, Kronman JH. A posteroanterior cephalometric evaluation of craniofacial asymmetry. Angle Orthod 1967;37(3):20511. 